\title{
Video-Assisted Thoracic Surgery Mediastinal Lymph Node Dissection in Lung Cancer Surgery
}

\author{
Kwhanmien Kim, M.D., Ph.D. \\ Department of Thoracic and Cardiovascular Surgery, Seoul National University Bundang Hospital, Seoul National University College of Medicine, Seongnam, Korea
}

\section{ARTICLE INFO}

Received June 1, 2021

Accepted July 16, 2021

Corresponding author

Kwhanmien Kim

Tel 82-31-787-7131

Fax 82-31-787-4050

E-mail kmkim0070@snubh.org

ORCID

https://orcid.org/0000-0002-6581-2750

\begin{abstract}
Mediastinal lymph node dissection is an important part of lung cancer surgery that provides accurate nodal staging and may improve survival outcomes. The minimally invasive approach, such as video-assisted thoracic surgery (VATS) lobectomy for patients with non-small cell lung cancer, has become a standard operation worldwide. VATS mediastinal lymph node dissection should be thorough and accurate to ensure the completeness of lung cancer surgery. Herein, the author describes techniques for VATS mediastinal lymph node dissection.
\end{abstract}

Keywords: Lung neoplasms, Video-assisted thoracic surgery, Lymph node excision

\section{Introduction}

Lymph node dissection, including the hilar and mediastinal lymph nodes, is an important procedure in non-small cell lung cancer surgery. Mediastinal lymph node dissection (MLND) is crucial for achieving complete surgery. Although whether MLND can increase survival has yet to be conclusively established, it can provide accurate pathological staging and better local control $[1,2]$. Complete lymph node dissection can be defined as the removal of all lymph nodes and fat tissues from each nodal station. The video-assisted thoracic surgery (VATS) lymph node dissection technique is almost the same as that of open thoracotomy, but it can vary according to the position of the thoracoscope, the presence of a working window, the surgical instruments used, and the adequacy of the surgical view provided by the thoracoscope. VATS MLND can now be safely performed and enables more lymph nodes to be harvested [3-9]. However, the extent of lymphadenectomy and the requirement of nodal stations for removal according to the lung cancer location have yet to be fully elucidated $[10,11]$.

\section{Port placement}

There are many different port placement techniques for VATS lung cancer surgery according to the surgeon's pref- erence. Before making VATS ports, it is important to consider a few points: an ergonomically good position to handle the instruments, ease of conversion to open thoracotomy in an emergency, a cosmetically favorable location, and the need to minimize postoperative pain. The author's standard VATS incisions include 2 ports and 1 utility incision (working window) [12]. The first port for the initial thoracoscopic exploration is placed at the seventh or eighth intercostal space (ICS) in the mid-axillary line. A working window of about $4 \mathrm{~cm}$ in length is placed at the fourth or fifth ICS in the mid-clavicular line. Specially-designed wound retractors are commercially available to enhance instrument handling and prevent wound contamination. An additional 5- or $10-\mathrm{mm}$ port is made at the fifth or sixth ICS below the scapular tip (Fig. 1). This port configuration guarantees safe and prompt conversion to open thoracotomy in emergent situations by connecting the working window and the posterior port. Simply by changing the thoracoscope port and applying appropriate maneuvers, all lung lobes can be resected and MLND can be achieved by this 3-port configuration without major modifications. However, the decisions of where to stand, where to introduce the thoracoscope, and whether to change the thoracoscope port or to use a fixed port all depend solely on the surgeon's preference. The most important consideration is to avoid competing with the assistant's instruments (the 
"no hand cross-over" technique). The author recommends always standing at the right side of the operating table because it allows smooth right-hand maneuvers. When performing left-side VATS lobectomy, a 10 -mm port for the thoracoscope is placed in the fifth or sixth ICS along the anterior scapular line, which can provide better fissure development and subcarinal exposure (Fig. 2).

\section{Techniques of VATS mediastinal lymph node díssection}

\section{Right side}

\section{Stations 2R, 3, and $4 R$}

The mediastinal pleura is opened from the junction of the azygos vein and the superior vena cava (SVC). From

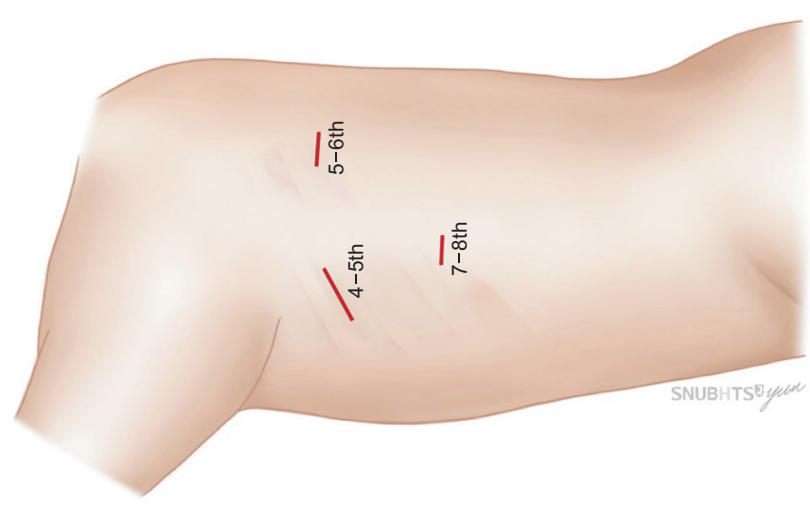

Fig. 1. Three port placement sites on the left side. below the azygos vein to the right brachiocephalic artery, between the trachea and the SVC, a longitudinal incision is made on the parietal pleura. It is easy to dissect all the mediastinal fat tissue, including the lymph nodes, from below the azygos vein to the level of the innominate artery in an upward direction. With elevation of the azygos vein and pushing down the right main pulmonary artery trunk, the mediastinal fat pad between the right main bronchus and anterior carinal area is dissected first, and then the dissection moves on to the pericardium around the aortic arch. The azygos vein is retracted caudally and the SVC is retracted anteriorly with an endoscopic retractor and peanut; the mediastinal fat pad is dissected from the trachea and the SVC and from the caudal border of the innominate artery (Fig. 3A, B). All mediastinal fat tissues, including the

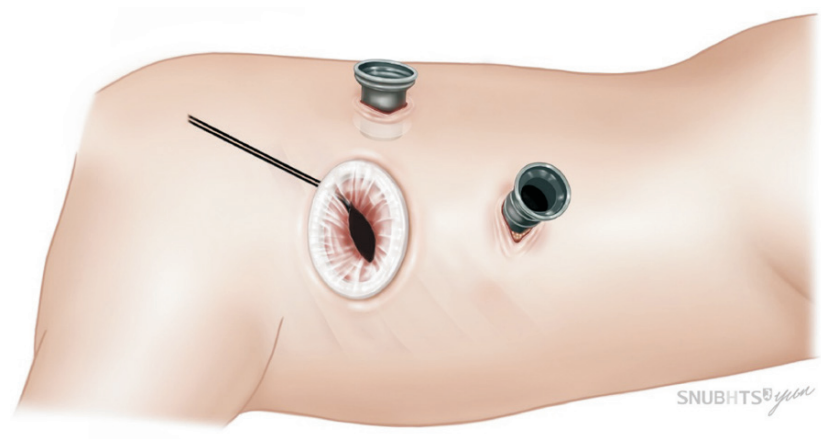

Fig. 2. Left-side 3-port placement with a 10-mm trocar in the fifth or sixth intercostal space along the anterior scapular line. If the thoracoscope is introduced through this back port, it can provide better fissure development and subcarinal exposure.
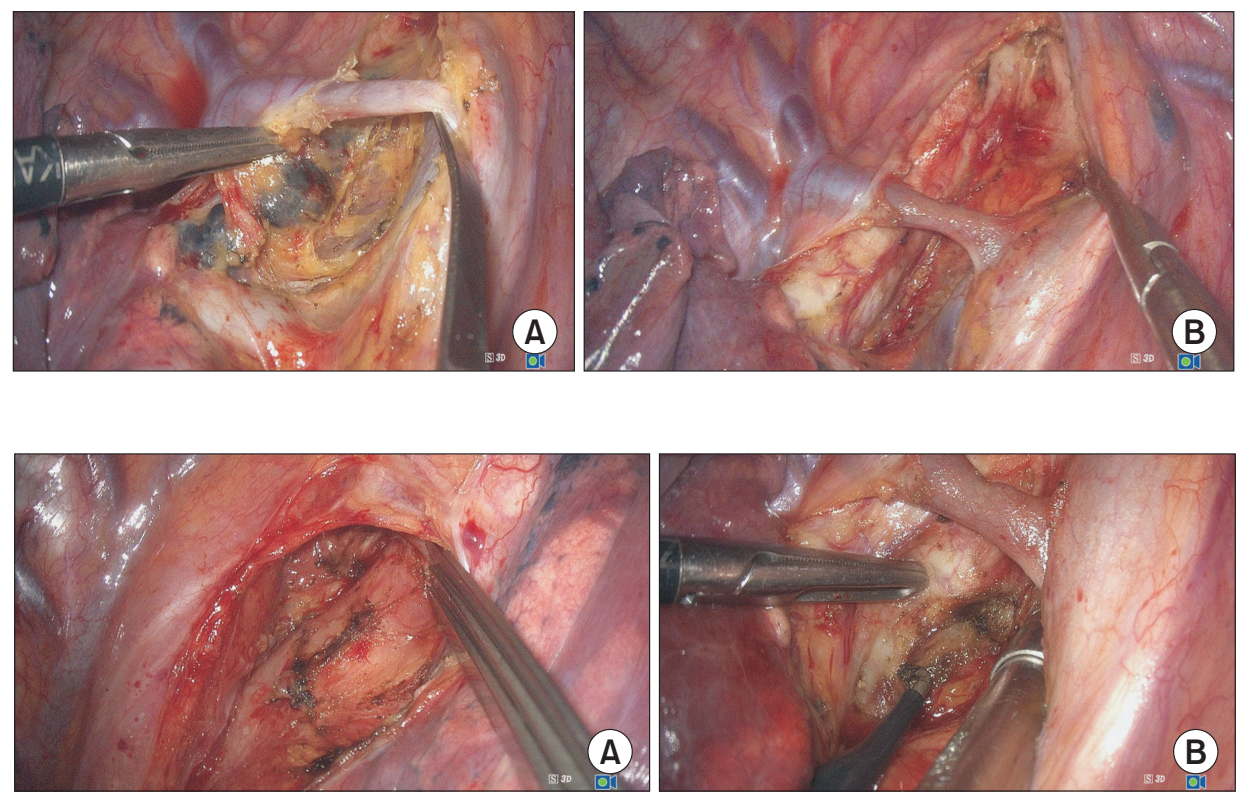

Fig. 4. (A) Right-side subcarinal lymph node dissection is performed while saving the vagus nerve pulmonary branches through the posterior approach. (B) The anterior subcarinal space can be reached through the anterior approach if clear dissection is difficult from behind.

Fig. 3. (A) Right upper mediastinal dissection starts from below the azygos vein, moving upward. (B) After comple nodection, the medicardium, the innominate artery, and the superior vena cava) can be seen. 
lymph nodes are removed en bloc. Small veins draining from the mediastinal fat tissues directly into the SVC are ligated with hemoclips or divided with an ultrasonic device. At the apex, the right recurrent laryngeal nerve can be damaged by thermal injury. Because there are many small lymphatic channels in this area, those should be ligated or treated safely to avoid postoperative chylothorax. The author prefers to use an ultrasonic device, because it is easy to dissect mediastinal fat tissues with less bleeding and it may help to prevent postoperative lymphatic leakage.

\section{Station 7 (right side)}

After tilting the operating table leftward and retracting the right lung anteriorly, the right subcarinal area is exposed easily. The parietal pleural is then opened, avoiding injury to the vagus nerve and its pulmonary branches. Although the small pulmonary vagal branches running into the bronchus are usually divided, it is better to save those branches to reduce pulmonary complications such as dry cough after operation. The subcarinal fat pad is dissected en bloc, starting inferiorly and moving upward to the cari-

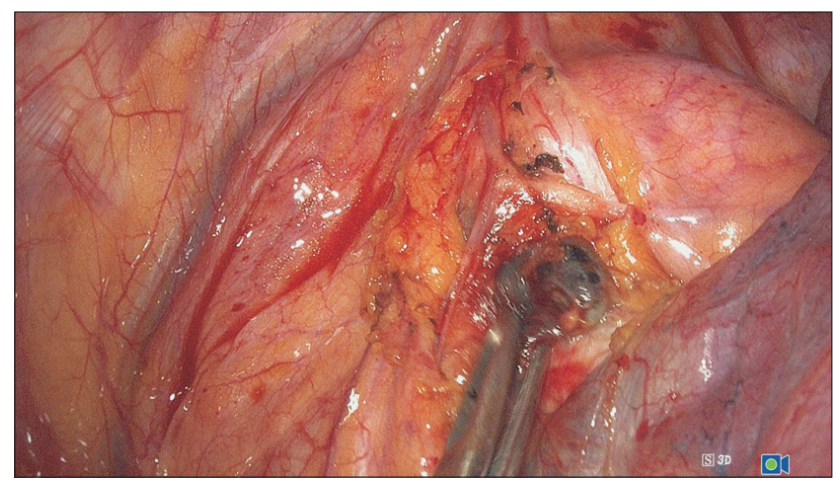

Fig. 5. Subaortic and paraaortic lymph nodes are dissected with care taken for the phrenic and left recurrent laryngeal nerves.

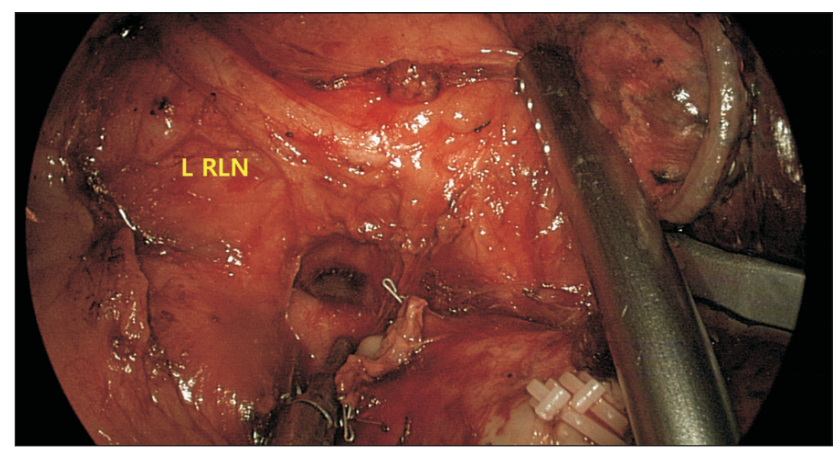

Fig. 6. The left recurrent laryngeal nerve (L RLN) is safely preserved after dissection of subaortic and left lower paratracheal lymph nodes. na with electrocautery or ultrasonic devices. Dissection starts from the inferior border of the bronchus intermedius and the inferior pulmonary vein, and then it moves upward. Then, subcarinal lymph nodes are dissected from the esophagus and the left and right main bronchi. The anterior subcarinal space can be reached by an anterior approach if clear dissection is difficult from behind (Fig. 4A, B).

\section{Left side}

\section{Stations 5 and 6}

The mediastinal pleura is opened between the phrenic

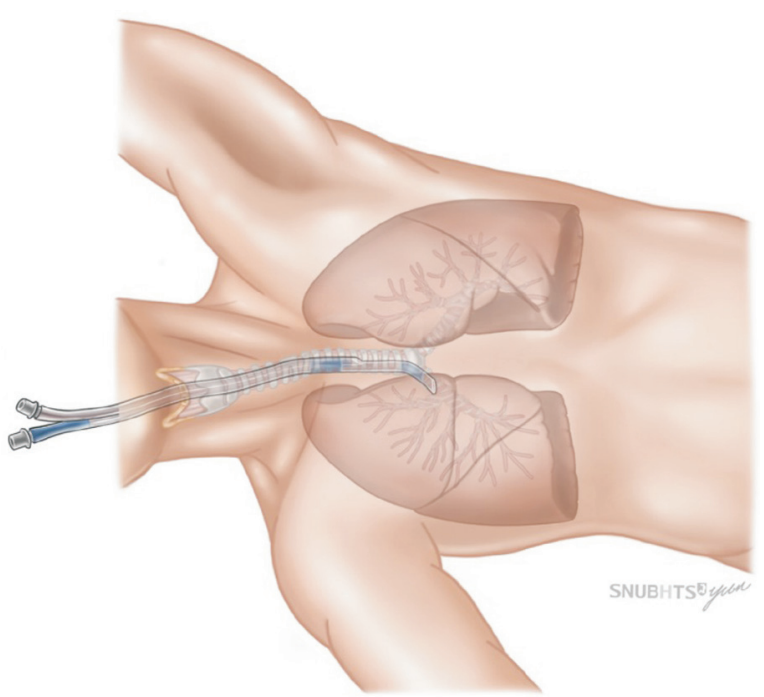

Fig. 7. Right-side double-lumen intubation can facilitate subcarinal lymph node dissection during left-side video-assisted thoracic surgery for lung cancer.

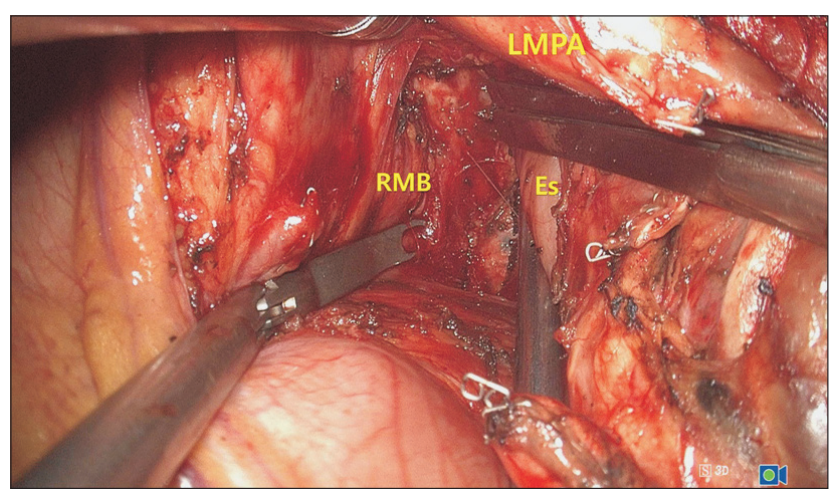

Fig. 8. The left subcarinal space can be approached anteriorly while elevating the left main bronchus and the left main pulmonary artery and pushing down the superior pulmonary vein stump, especially after left upper lobectomy. RMB, right main bronchus; LMPA, left main pulmonary artery; Es, esophagus. 
and the vagus nerves at the level of the aortopulmonary window. The incision is extended along the vagus nerve in a cephalad direction and horizontally above the left main pulmonary artery. Attention should be paid to avoid the phrenic nerve and left recurrent laryngeal nerve injuries. The level 6 lymph nodes are located in the area medial to the ligamentum arteriosum and the level 5 lymph nodes are between the lower border of the aortic arch and the upper rim of the left main pulmonary artery. The aortopulmonary fat pad containing the level 5 and 6 lymph nodes is removed en bloc (Fig. 5).

\section{Station $4 \mathrm{~L}$}

The left paratracheal lymph nodes are located between the upper margin of the aortic arch and the upper rim of the left main pulmonary artery. After pushing down the left main pulmonary artery with a peanut and retracting the aortic arch upward with an endoscopic retractor, the $4 \mathrm{~L}$ lymph nodes are dissected along the left main bronchus to the trachea. The $4 \mathrm{~L}$ lymph nodes are close to the left recurrent laryngeal nerve. To avoid left vocal cord palsy, the left recurrent laryngeal nerve and the proximal vagus nerve must be carefully preserved (Fig. 6).

\section{Station 7 (left side)}

It is a little more difficult to expose the left subcarinal space because of the longer left main bronchus, the descending aorta, and the spine. A right-sided double-lumen endotracheal intubation is necessary to facilitate the elevation of the left main bronchus during the left subcarinal lymph node dissection (Fig. 7). The left subcarinal space can be approached anteriorly with the lung retracted posteriorly and elevating the left main bronchus and the left main pulmonary artery, especially after left upper lobectomy (Fig. 8). When the left lower lobectomy is performed, the left subcarinal space is approached posteriorly. The mediastinal pleura is opened anterior to and along the descending aorta. The small branches of the vagus nerve supplying the lung are divided. To expose the left subcarinal space, the left main bronchus is retracted anteriorly with an endoscopic fan retractor or endoscopic peanut, and the descending aorta and the esophagus are retracted posteriorly with an endoscopic esophageal retractor. The subcarinal fat pad is dissected from the left main bronchus to the carina. Lymph node dissection is then carried out from the esophagus and the right main bronchus. The small arterial branches entering the lymph nodes from the anterior border of the trachea at the level of the carina should be treated properly to avoid postoperative bleeding.

\section{Conclusion}

VATS mediastinal lymph node dissection is essential part of lung cancer surgery and can be performed completely with appropriate surgical techniques.

\section{Conflict of interest}

No potential conflict of interest relevant to this article was reported.

\section{Acknowledgments}

I would like to thank Nayun Lee for drawing the figures (Figs. 1, 2, 7) in this manuscript.

\section{ORCID}

Kwhanmien Kim: https://orcid.org/0000-0002-6581-2750

\section{References}

1. Keller SM, Adak S, Wagner H, Johnson DH. Mediastinal lymph node dissection improves survival in patients with stages II and IIIa non-small cell lung cancer. Ann Thorac Surg 2000;70:358-65.

2. Allen MS, Darling GE, Pechet TT, et al. Morbidity and mortality of major pulmonary resections in patients with early-stage lung cancer: initial results of the randomized, prospective ACOSOG Z0030 trial. Ann Thorac Surg 2006;81:1013-9.

3. Watanabe A, Koyanagi T, Ohsawa H, et al. Systematic node dissection by VATS is not inferior to that through an open thoracotomy: a comparative clinicopathologic retrospective study. Surgery 2005; 138:510-7.

4. Sagawa M, Sato M, Sakurada A, et al. A prospective trial of systematic nodal dissection for lung cancer by video-assisted thoracic surgery: can it be perfect? Ann Thorac Surg 2002;73:900-4.

5. Palade E, Passlick B, Osei-Agyemang T, Gunter J, Wiesemann S. Video-assisted vs open mediastinal lymphadenectomy for stage I non-small-cell lung cancer: results of a prospective randomized trial. Eur J Cardiothorac Surg 2013;44:244-9.

6. Lee HS, Jang HJ. Thoracoscopic mediastinal lymph node dissection for lung cancer. Semin Thorac Cardiovasc Surg 2012;24:131-41.

7. Kim K, Kim HK, Park JS, et al. Video-assisted thoracic surgery lobectomy: single institutional experience with 704 cases. Ann Thorac Surg 2010;89:S2118-22.

8. Kim HK, Choi YS, Kim J, Shim YM, Kim K. Outcomes of unexpected pathologic N1 and N2 disease after video-assisted thoracic surgery lobectomy for clinical stage I non-small cell lung cancer. J Thorac Cardiovasc Surg 2010;140:1288-93. 
9. Amer K. Thoracoscopic mediastinal lymph node dissection for lung cancer. Semin Thorac Cardiovasc Surg 2012;24:74-8.

10. Shapiro M, Kadakia S, Lim J, et al. Lobe-specific mediastinal nodal dissection is sufficient during lobectomy by video-assisted thoracic surgery or thoracotomy for early-stage lung cancer. Chest 2013;144: 1615-21.

11. Ishiguro F, Matsuo K, Fukui T, Mori S, Hatooka S, Mitsudomi T. Effect of selective lymph node dissection based on patterns of lobe-spe- cific lymph node metastases on patient outcome in patients with resectable non-small cell lung cancer: a large-scale retrospective cohort study applying a propensity score. J Thorac Cardiovasc Surg 2010; 139:1001-6.

12. Kim K. VATS mediastinal lymph node dissection. In: Scarci M, Solli P, Sihoe AD, editors. Minimally invasive thoracic surgery: principles and practice of thoracoscopic technique. London: JP Medical Publishers; 2017. p. 37-40. 